\title{
On external semi-global stochastic stabilization of a double integrator with input saturation
}

\author{
Anton A. Stoorvogel ${ }^{1}$, Ali Saberi ${ }^{2}$
}

\begin{abstract}
This paper considers external semi-global stochastic stabilization for linear plants with saturating actuators, driven by a stochastic external disturbance, and having random Gaussian-distributed initial conditions. Recently, it has been shown that for criticaly stable systems there exists a linear static state feedback law that achieves global asymptotic stability in the absence of disturbances, while guaranteeing a bounded variance of the state vector in the presence of disturbances and Gaussian distributed initial conditions. We report how this result extends to a double integrator with input saturation
\end{abstract}

\section{INTRODUCTION}

Internal and external stabilization of linear plants with actuators subject to saturation has been the subject of intense renewed interest among the control research community for the past two decades. The number of recent books and special issues of control journals devoted to this subject matter evidence this intense research focus, see for instance [1], [4], [10], [13] and the references therein. It is now considered a classical fact that both continuous- and discrete-time linear plants with saturating actuators can be globally internally stabilized if and only if all of the open-loop poles are located in the closed left half plane (in the continuous-time case), and are in inside and/or on the unit circle (in the discrete-time case). These conditions on open-loop plants can be equivalently stated as a requirement that the plant be asymptotically null controllable with bounded control (ANCBC). It is also a classical fact that, in general, global internal stabilization of ANCBC

\footnotetext{
${ }^{1}$ Department of Electrical Engineering, Mathematics and Computer Science, University of Twente, P.O. Box 217, Enschede, The Netherlands. E-mail: A.A.Stoorvogel eutwente.nl.

${ }^{2}$ School of Electrical Engineering and Computer Science, Washington State University, Pullman, WA 99164-2752, U.S.A. E-mail: saberi@eecs.wsu.edu .
}

plants requires nonlinear feedback laws. See for instance. [2], [9], [14].

By weakening the notion of global internal stabilization to a semiglobal framework, Saberi et al. showed that ANCBC plants with saturating actuators can be internally stabilized in the semiglobal sense using only linear feedback laws. Such a relaxation from a global to semiglobal framework is, from an engineering standpoint, both sensible and attractive. A body of work exists proposing design methodologies (low-gain, low-high gain, and variations) for both continuous- and discretetime ANCBC plants with saturating actuators. See for instance [3], [5], [6], [15].

With regard to external stabilization, the picture is complicated. Unlike linear plants, internal stability does not necessarily guarantee external stability when saturation is present. Hence, stabilization must be done simultaneously in both the internal and external sense. There is a body of work that examines the standard notion of $L_{p}$ stability for ANCBC plants with saturating actuators when the external input (disturbance) is input-additive and at the same time requiring either global or semi global internal stability in the absence of disturbance, see [8]. For the more general case in which the disturbance is not necessarily input-additive, there are surprising results in the literature which point to the complexity of the notion of external stability for ANCBC plants with saturating actuators. Notable among these is the result from [11] of Stoorvogel et.al., that for a double integrator with saturating actuators, "any linear internally stabilizing control law achieves $L_{p}$ stability without finite gain for $p \in[1,2]$, but no linear internally stabilizing control law can achieve $L_{p}$ stability for $p \in(2, \infty]$ ". All these considerations lead us to believe that a suitable notion of external stability for linear plants with saturating actuators, and in- 
deed for general nonlinear systems in the presence of disturbances and nonzero initial conditions, is yet to be developed.

In this paper we look at the simultaneous external and internal stabilization of ANCBC plants with saturating actuators when the external input is a stochastic disturbance. In a previous paper [12] we established that for critically stable systems, for any given variance of the external disturbance, there exists a linear feedback which achieves global internal asymptotic stability and, moreover, guarantees a bounded variance of the state. The remaining question is what happens for critically unstable systems

In this paper we consider a double integrator subject to input saturation, stochastic external disturbances. It is known that any linear controller which stabilizes the system when ignoring the saturation also achieves global asymptotic stability when the saturation is present. In other words, the saturation element can never destabilize the system when considering internal stability. However, this paper will prove that for any linear controller the variance of the state will be unbounded for sufficiently large variance of the external disturbance. On the other hand, any linear controller achieves a bounded variance of the state for small enough variance of the external disturbance. Moreover, for any given variance of the external disturbance we can find a linear controller which will achieve a bounded variance for the state.

\section{PROBlem FORMUlation AND MAIN RESULTS}

We consider the stochastic differential equation

$$
\mathrm{d} x(t)=A x(t) \mathrm{d} t+B \sigma(u(t)) \mathrm{d} t+E \mathrm{~d} w(t)
$$

where the state $x$, the control $u$ and the disturbance $w$ are vector-valued signals of dimension $n, m$ and $\ell$, respectively. Here $w$ is a Wiener process (a process of $\ell$ independent Brownian motions) with mean 0 and rate $Q$, that is, $\operatorname{Var}[w(t)]=Q t$ and the initial condition $x_{0}$ of (1) is a Gaussian random vector which is independent of $w$. Its solution $x$ is rigorously defined through Wiener integrals and is a Gauss-Markov process. See, for instance, [7]. Moreover $\sigma$ is the standard saturation function given by:

$$
\sigma(u)= \begin{cases}-1 & u<-1 \\ u & -1 \leq u \leq 1 \\ 1 & u>1\end{cases}
$$

and admissible feedbacks are possibly nonlinear static state feedbacks of the form

$$
u(k)=f(x(k))
$$

where $f$ is a Lipschitz-continuous mapping with $f(0)=0$.

Problem II.1 Given the system (1), the semiglobal external stochastic stabilization problem is to find an admissible feedback (2) such that the following properties hold:

(i) in the absence of the external input $w$, the equilibrium point $x=0$ of the controlled system (1)-(2) is globally asymptotically stable.

(ii) the variance $\operatorname{Var}(x(t))$ of the state of the controlled system (1)-(2) is bounded over $t \geq 0$.

As a follow-up on our previous work in [12], we consider the double-integrator which has played an important role in obtaining insight in what is possible or not given the presence of an input saturation:

$$
\begin{aligned}
& \mathrm{d} x_{1}(t)=x_{2}(t) \mathrm{d} t+E_{1} \mathrm{~d} w(t) \\
& \mathrm{d} x_{2}(t)=\sigma(u(t)) \mathrm{d} t+E_{2} \mathrm{~d} w(t)
\end{aligned}
$$

It is well-known that any linear controller which asymptotically stabilizes the linear system above without the saturation element will still internally stabilize the system with the saturation function present, i.e. any controller of the form:

$$
u=-k_{1} x_{1}-k_{2} x_{2}
$$

will achieve global internal stability (when $w \equiv 0$ ) provided $k_{1}>0$ and $k_{2}>0$. This can be easily verified using the Lyapunov function

$$
V\left(x_{1}, x_{2}\right)=\int_{0}^{-k_{1} x_{1}-k_{2} x_{2}} \sigma(\xi) \mathrm{d} \xi+\frac{k_{1}}{2} x_{2}^{\mathrm{T}} x_{2} .
$$

Theorem II.2 Consider the system (3).

Any linear feedback of the form (4) will result in an unbounded variance of the state for $Q$ large enough whenever $E_{2} \neq 0$ 
In other words, the above theorem clearly states that global internal stability does not imply global external stability.

Theorem II.3 Consider the system (3).

Any linear feedback of the form (4) will result in a bounded variance of the state for $Q$ small enough.

Moreover, for any given $Q$, there exists a controller of the form (4) which will result in a bounded variance of the state.

Our proof will actually establish that for any given $Q$ we can guarantee that all moments of the state are bounded in time. Note that, like in the critically stable case, the tail of the distribution of the state will be slower than in a Gaussian distribution (actually for it is comparable with the tail of an exponential distribution). This is actually logical since we can not guarantee a sufficient decay when the state gets big due to the saturation. We actually get a linear decay compared to an exponential decay.

\section{PROOF OF THE MAIN RESUlTS}

Proof of Theorem II.2 : We consider the system (3) with controller (4). We first use a change of variables

$$
\begin{aligned}
& y_{1}(\tau)=k_{1} x_{1}\left(\frac{k_{2}}{k_{1}} \tau\right)+k_{2} x_{2}\left(\frac{k_{2}}{k_{1}} \tau\right) \\
& y_{2}(\tau)=k_{2} x_{2}\left(\frac{k_{2}}{k_{1}} \tau\right)
\end{aligned}
$$

while

$$
\tilde{w}(\tau)=\sqrt{\frac{k_{1}}{k_{2}}} w\left(\frac{k_{2}}{k_{1}} \tau\right)
$$

We note that $\tilde{w}$ (like $w$ ) is Wiener process with mean 0 and rate $Q$. We obtain:

$$
\begin{aligned}
& \mathrm{d} y_{1}(\tau)=y_{2}(\tau) \mathrm{d} \tau-\lambda \sigma\left(y_{1}(\tau)\right) \mathrm{d} \tau+\tilde{E}_{1} \mathrm{~d} \tilde{w}(\tau) \\
& \mathrm{d} y_{2}(\tau)=-\lambda \sigma\left(y_{1}(\tau)\right) \mathrm{d} \tau+\tilde{E}_{2} \mathrm{~d} \tilde{w}(\tau)
\end{aligned}
$$

where

$$
\lambda=\frac{k_{2}^{2}}{k_{1}}
$$

and

$$
\tilde{E}_{1}=\sqrt{\frac{k_{2}}{k_{1}}}\left(k_{1} E_{1}+k_{2} E_{2}\right), \quad \tilde{E}_{2}=\sqrt{\frac{k_{2}}{k_{1}}} k_{2} E_{2} .
$$

Next, we look at the Lyapunov function $V$ defined in (5) which in our new coordinates looks like:

$$
V\left(y_{1}, y_{2}\right)=\int_{0}^{y_{1}} \sigma(\xi) \mathrm{d} \xi+\frac{1}{2 \lambda} y_{2}^{\mathrm{T}} y_{2} .
$$

We define $y=\left(y_{1}, y_{2}\right)$.

We consider two cases. If $\left|y_{1}\right| \leq 1$ we find that:

$$
\begin{array}{r}
d V=-\lambda \sigma\left(y_{1}\right)^{2} \mathrm{~d} \tau+\left[\sigma\left(y_{1}\right) \tilde{E}_{1}+\frac{1}{\lambda} y_{2} \tilde{E}_{2}\right] \mathrm{d} \tilde{w} \\
+\left[\tilde{E}_{1} Q \tilde{E}_{1}^{\prime}+\frac{1}{\lambda} \tilde{E}_{2} Q \tilde{E}_{2}^{\prime}\right] \mathrm{d} \tau
\end{array}
$$

while for $\left|y_{1}\right|>1$ we get:

$$
\begin{array}{r}
d V=-\lambda \sigma\left(y_{1}\right)^{2} \mathrm{~d} \tau+\left[\sigma\left(y_{1}\right) \tilde{E}_{1}+\frac{1}{\lambda} y_{2} \tilde{E}_{2}\right] \mathrm{d} \tilde{w} \\
+\frac{1}{\lambda} \tilde{E}_{2} Q \tilde{E}_{2}^{\prime} \mathrm{d} \tau
\end{array}
$$

Note that in the above two equations the last term is the special correction term that comes from Itô's lemma. For details we refer to [7].

Combining the above, we find the following lower bound:

$$
\begin{aligned}
\mathbb{E}[V(y(t))] \geq \mathbb{E}\left[V\left(y\left(t_{0}\right)\right)\right] & -\int_{t_{0}}^{t} \lambda \mathrm{d} \tau \\
& +\int_{t_{0}}^{t} \frac{1}{\lambda} \tilde{E}_{2} Q \tilde{E}_{2}^{\prime} \mathrm{d} \tau
\end{aligned}
$$

It is clear that $V(y) \leq\|y\|^{2}$. Using the above lower bound we find $\operatorname{Var}[y]$ is unbounded whenever:

$$
\tilde{E}_{2} Q \tilde{E}_{2}^{\prime} \geq \lambda^{2}
$$

Here we used that $\operatorname{Var}[y]=\mathbb{E}\|y\|^{2}$ given that $y$ has mean zero. The above clearly means that for any given controller there exist stochastic disturbances with sufficiently large variance that will result in an unbounded variance for the state. The only possible exception is when $E_{2}=0$. Finally note that if the variance of $y$ is unbounded then we clearly also have that the variance of $x$ is unbounded.

Outline of the proof of Theorem II.3 : We use the same notation as in the proof of Theorem II.2. Considering (8) and (9), we find that $V(t)$ can never be a supermartingale since close to the origin we will have $\mathbb{E}\left[V\left(y\left(t_{1}\right) \mid V\left(y\left(t_{0}\right)\right)\right] \geq V\left(y\left(t_{0}\right)\right)\right.$. Moreover, establishing that $\mathbb{E}[V(t)]$ is bounded is not sufficient to establish that the variance of $y$ is bounded since, for large $y_{1}, V$ is only linear in $y_{1}$. Instead, we will establish that for suitable $R$ and $\gamma$, we have that:

$$
\mathbb{E}[V(y(k)) \geq M] \leq R e^{-\gamma \sqrt{M}}
$$

for $k \in \mathbb{Z}$. This is shown by establishing that it is true for $k=j$ if it is true for $k=j-1$. From 
the Gaussian initial conditions it is clear that it is true for $k=0$. The motivation for working with $t=k(k \in \mathbb{Z})$ has to with the fact that if $V$ is large at any given moment but $y_{1}$ is small than we can have an increase of $V$. But $V$ large while $y_{1}$ small guarantees that $y_{2}$ is large and hence $y_{1}$ will only be small for a brief period of time and hence over a period of a second we can still guarantee the required behavior. Finally in order to establish (10) for $k=j$ we find a relationship between $\lambda$ and $Q$ which shows that for any $\lambda$ the recursion is valid for $Q$ small enough and moreover for any $Q$ there exists $\lambda$ large enough for which the recursion is valid.

Next we note that if (10) is satisfied for all $k \in \mathbb{Z}$ then we have that for any $m$ :

$$
\mathbb{E} V^{m}(y(k))
$$

is bounded. But this implies that all moments of $y(k)$ are bounded. For instance

$$
y_{1}^{2} \leq 2\left(\int_{0}^{y_{1}} \sigma(s) \mathrm{d} s\right)^{2}<2 V^{2}
$$

and

$$
y_{2}^{2} \leq 2 \lambda V
$$

Therefore

$$
\|y(k)\|^{2} \leq 2 V^{2}(y(k))+2 \lambda V(y(k))
$$

and we find that since $\mathbb{E} V(y(k))$ and $\mathbb{E} V^{2}(y(k))$ are bounded that the variance of $y(k)$ is bounded as well.

Finally, it is easily verified that $V(y(t))$ will have bounded moments for $t \in \mathbb{R}$ whenever $V(y(k))$ will have bounded moments for $k \in \mathbb{Z}$.

\section{Discussion AND CONCLUSIONS}

This paper is the continuation of our earlier efforts toward understanding and formulating the notion of simultaneous external and internal stabilization for linear plants with saturating actuators. Here we have studied what is possible for the canonical example of a double integrator with a linear state feedback law. We noted that global asymptotic stability of the closed-loop system in the absence of disturbances, does not guarantee bounded variance of the state vector for all time in the presence of disturbances. However, this result is true for disturbances with small enough variance. Finally, we showed that for any given variance we can design a controller which achieves a bounded variance of the state. We conjecture that the above can be expanded to arbitrary linear systems with instabilities which grow at most linearly. However, whether the same will be true for arbitrary controllable linear systems with all eigenvalues in the closed left half plane remains as a major open problem.

\section{REFERENCES}

[1] D.S. Bernstein And A.N. Michel, Guest Eds., Special Issue on saturating actuators, Int. J. Robust \& Nonlinear Control, 5(5), 1995, pp. 375-540.

[2] A.T. FULLER, "In-the-large stability of relay and saturating control systems with linear controller", Int. J. Contr., 10(4), 1969, pp. 457-480.

[3] P. Hou, A. Saberi, Z. Lin, and P. Sannuti, "Simultaneously external and internal stabilization for continuous and discrete-time critically unstable systems with saturating actuators", Automatica, 34(12), 1998, pp. 1547-1557.

[4] V. Kapila And G. GRIGORIADIS, Eds., Actuator saturation control, Marcel Dekker, 2002.

[5] Z. LIN AND A. SABERI, "Semi-global exponential stabilization of linear discrete-time systems subject to "input saturation' via linear feedbacks”, Syst. \& Contr. Letters, 24, 1995, pp. 125-132.

[6] Z. Lin, A. SABeri, AND A.A. Stoorvogel, "Semi-global stabilization of linear discrete-time systems subject to input saturation via linear feedback - an ARE-based approach", IEEE Trans. Aut. Contr., 41(8), 1996, pp. 1203-1207.

[7] B. ØKSENDAL, Stochastic differential equations: an introduction with applications, Universitext, Springer-Verlag, Berlin, Sixth Ed., 2003.

[8] A. Saberi, P. Hou, And A.A. Stoorvogel, "On simultaneous global external and global internal stabilization of critically unstable linear systems with saturating actuators", IEEE Trans. Aut. Contr., 45(6), 2000, pp. 1042-1052.

[9] E.D. Sontag AND H.J. SuSsmanN, "Nonlinear output feedback design for linear systems with saturating controls", in Proc. 29th CDC, Honolulu, 1990, pp. 3414-3416.

[10] A. Saberi And A.A. Stoorvogel, Guest Eds., Special Issue on control problems with constraints, Int. J. Robust \& Nonlinear Control, 9(10), 1999, pp. 583-734.

[11] A.A. Stoorvogel, G. Shi, ANd A. SAberi, "External stability of a double integrator with saturated linear control laws", Dynamics of Continuous Discrete and Impulsive Systems, Series B: Applications \& Algorithms, 11(4-5), 2004, pp. $429-451$.

[12] A.A. Stoorvogel, S. Weiland, and A. Saberi, "On external semi-global stochastic stabilization of linear systems with input saturation", in American Control Conference, New York, 2007, pp. 5845-5850. To appear in IEEE Trans. Aut. Control.

[13] S. Tarbouriech and G. Garcia, Eds., Control of uncertain systems with bounded inputs, vol. 227 of Lecture notes in control and information sciences, Springer Verlag, 1997. 
[14] A.R. TEEL, "Global stabilization and restricted tracking for multiple integrators with bounded controls", Syst. \& Contr. Letters, 18(3), 1992, pp. 165-171.

[15] Y. YANG, Global stabilization of linear systems with bounded feedback, PhD thesis, Rutgers University, New Brunswick, 1993. 